\title{
Four-port coupled channel-guide device based on 2D photonic crystal structure
}

\author{
Edilson A. Camargo ${ }^{\text {a,b,*, Harold M.H. Chong }}{ }^{\text {, }}$, Richard M. De La Rue \\ ${ }^{\mathrm{a}}$ Optoelectronics Research Group, Department of Electronics and Electrical Engineering, University of Glasgow, \\ Oakfield Avenue, Glasgow G12 8LT, Scotland, UK \\ ${ }^{\mathrm{b}}$ Instituto Tecnológico de Aeronáutica, Centro Técnico Aeroespacial, Praca Marechal Eduardo Gomes, \\ 50 São José dos Campos, 12228-901 São Paulo, Brazil
}

Received 6 October 2004; received in revised form 22 November 2004; accepted 22 November 2004

Available online 28 November 2004

\begin{abstract}
We have fabricated and measured a four-port coupled channel-waveguide device using W1 channel waveguides oriented along $\Gamma K$ directions in a two-dimensional (2D) hole-based planar photonic crystal (PhC) based on silicon-on-insulator (SOI) waveguide material, at operation wavelengths around $1550 \mathrm{~nm}$. 2D FDTD simulations and experimental results are shown and compared. The structure has been designed using a mode conversion approach, combined with coupled-mode concepts. The overall length of the photonic crystal structure is typically about $39 \mu \mathrm{m}$ and the structure has been fabricated using a combination of direct-write electron-beam lithography (EBL) and dry-etch processing. Devices were measured using a tunable laser with end-fire coupling into the planar structure.
\end{abstract}

(C) 2004 Published by Elsevier B.V.

PACS: $42.70 . \mathrm{Q} ; 42.79 . \mathrm{F}$

Keywords: Photonic crystals; Four-port devices; Mode conversion; Couplers

\section{Introduction}

The four-port coupler photonic crystal (PhC) channel-guide device is a potentially important component for optical integrated circuits, since it allows light to cross (or not) between different

\footnotetext{
* Corresponding author. Tel.: +44 141330 8631; fax: +441413304907.

E-mail address: camargo@elec.gla.ac.uk (E.A. Camargo).
}

waveguides within a limited space. Ideally, light launched into one of the input waveguides can be fully transmitted into an alternative output waveguide using the coupling region, without any power remaining in the straight through waveguide and with negligible reflection and radiation losses.

The device design described in this paper is based on high but variable filling factor PhC structures being used to tune the modes and enhance the transmission [1], with shifted holes in the corners of the bend 
regions [2,3], as presented by the authors in previous work [4-6], combined with the concept of $\mathrm{PhC}$ channel-to-channel directional couplers, as presented by Thorhauge et al. [7]. The design of $\mathrm{PhC}$ directional couplers using a simple reversed Y-junction arrangement was presented in [8] and showed that, in this type of structure, a significant amount of the optical beam maybe scattered into the other input port of the Yjunction, rather than reach the coupled waveguide section and also the output ports. Additionally, the concept of directional coupling has been used to develop wavelength selective optical filters, as reported in $[9,10]$, with strong wavelength dependence.

\subsection{The coupling mechanism}

The coupling process is based on the coupled-mode approach [11] - in which light travelling in one of the waveguides can be described as a superposition of two fundamental modes of the directional coupler, one even and one odd (in the simplest case).

At certain propagation lengths the phase shift $(\phi)$ between the two modes (even and odd) launched in the coupler region via the input waveguides differs by an odd integer multiple of $\pi$, the switching condition.

$$
\begin{gathered}
\left|\phi_{\text {even }}-\phi_{\text {odd }}\right|=(2 m+1) \pi, \\
m=0.1,2.3, \ldots,
\end{gathered}
$$

At this point, when the two modes are correctly synchronized, the guided optical power is maximally transferred (totally in the ideal case) from the direct channel-waveguide side of the coupler into the other waveguide. The complete transfer of energy takes place periodically in the direction of the propagation with a period given by the coupling length $L \pi(m)$ and the distance required for the coupling process, determined by the coupling coefficient $(\kappa)$, is obtained by:

$L_{\pi}=\frac{m \pi}{2 \kappa}, \quad$ for integer values of $m$

According to the classical approach of the coupledmode theory [11] for the two dielectric waveguides with good modal confinement, which is, we believe, still appropriate for the case of the $\mathrm{PhC}$ channel-guide coupler, the calculation of the coupling between the waveguides often centres on an evaluation of the coupling coefficient $\kappa$ [12], which may be expressed as:

$\kappa=\frac{2 k_{\mathrm{g}}^{2} k_{\mathrm{L}} \mathrm{e}^{-k_{\mathrm{L}} s}}{2 \beta w\left(k_{\mathrm{L}}^{2}+k_{\mathrm{g}}^{2}\right)}$

where the $k_{\mathrm{g}}$ and $k_{\mathrm{L}}$ terms are the transverse propagation constants in the waveguide and the exterior decay constants, respectively, $\beta$ is the modal propagation constant, $w$ is the width of the channel waveguides and $s$ is the spacing between the two waveguides. The strong (exponential) dependence on the spacing can explain the strong influence of the size of the holes in the coupling region.

The value of the coupling coefficient $(\kappa)$ can also be tuned properly by changing the radius of the central row of holes between the waveguides [13] or, more generally, by changing the effective index of refraction of the region between waveguides, as was previously stated in [14]. The size of the holes in the coupling region should be smaller than that of the holes in the rest of the structure because the difference in phase-velocity between the modes, at a given frequency, is directly dependent on the distance between the waveguides which is given by the size of the holes in the coupling region. Based on numerical simulation of the propagation in the coupler region, it was found that the length of the coupling region of the device should be $27 a$ $(11.34 \mu \mathrm{m})$, for our particular design, where " $a$ " is the lattice pitch of $420 \mathrm{~nm}$ used in our experiments at wavelengths around $1550 \mathrm{~nm}$.

\section{Design and simulation}

The device design is shown in Fig. 1 and consists of two input $\mathrm{W} 1 \mathrm{PhC}$ channel waveguides connected to two outputs $\mathrm{W} 1 \mathrm{PhC}$ channel waveguides through a coupling region. The coupling region consists of two $\mathrm{PhC}$ channel waveguides placed next to each other and separated by a single row of coupling holes with reduced radius ( $20 \%$ of the periodicity).

In order to obtain stronger coupling of the modes between the two integrated components of the full device (the Y-junction and the coupler section), the Yjunction design has required some modification. The concept of adiabatic mode transformation, with the technique of tapering of the holes in the $\mathrm{PhC}$ channel 


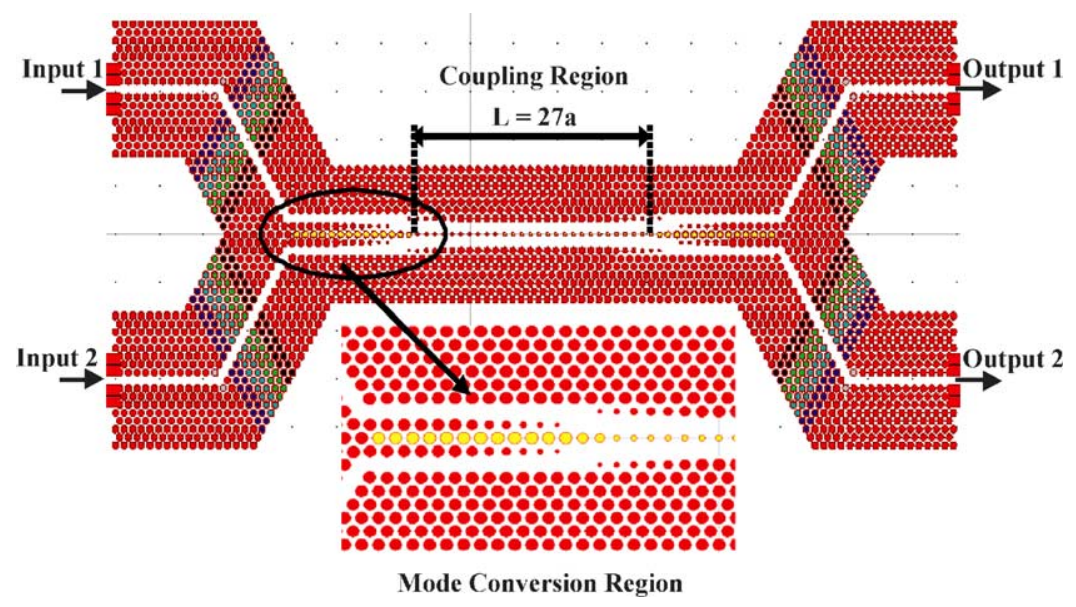

Fig. 1. The four-port device formed by two modified beam splitters connected by a 27 period directional coupler, with smaller holes in the coupling region. Details of the modified splitter using a mode conversion region are also shown.

waveguides, as presented in $[15,16]$, has been used. The taper is formed by a series of holes in which the feature dimensions vary progressively.

This progressive adaptation requires the size of the holes to have dimensions smaller than those of the holes forming the $\mathrm{PhC}$ channel waveguide. In this way the taper implements a form of artificial material effective-index gradient, permitting an independent optimisation of the mode profile for effective and smooth input and output coupling, thereby, increase the transmission efficiency of the device. The device design presented in this paper uses the tapering technique to couple modes from the $\mathrm{PhC}$ beam splitter into the coupler region and vice versa.

The PhC splitter has a Y-junction modified by using a three rows of holes separation in order to avoid coupling of the modes due to resonant cavity effects in the junction region [8]. The channel waveguides emerging from the Y-junction are then tapered down towards the interface with the waveguide coupler region, which also has hole sizes that taper upwards on the outside edges of the channels, as can be seen in Fig. 1.

The above conceptual approach is quite similar to the design presented in [17], which was possibly the first use of this approach in the design of tapered $\mathrm{PhC}$ channel waveguides, in order to couple light from W3 to $\mathrm{W} 1 \mathrm{PhC}$ channel waveguides-giving a coupling efficiency of about $50 \%$. The same approach was also used in W3 channel waveguide bends to reduce the width of the corner, improving the modal propagation and increasing the transmission efficiency [18].

The general parameters of the four-port $\mathrm{PhC}$ device structure for $1550 \mathrm{~nm}$ operation were: period $=420 \mathrm{~nm}$, taper hole radius starting from $60 \mathrm{~nm}$ and increasing to $150 \mathrm{~nm}$ in steps of $20 \mathrm{~nm}$ (the last step was only $10 \mathrm{~nm}$ in order to provide the correct designed filling factor), distributed along six periods on the opposite sides of the channel waveguide interface.

The radius of the 'standard' holes in the whole structure was about $165 \mathrm{~nm}$, giving a filling factor of $50 \%$. The structure also had shifted holes in the bend regions, as mentioned previously, and had a total length of $40 \mu \mathrm{m}$.

The 2D FDTD simulated transmissions of field spectral characteristics for the four-port device are shown in Fig. 2 with light launched through input port 1 with the PML boundary conditions set by the software. The effective index used in the simulation was 3.28. The transmitted light into the output ports is shown, with port 1 shown as a dashed line and port 2 as a bold line. The high transmission region for the coupled-across output, port 2, is between 1480 and $1535 \mathrm{~nm}$, giving a total bandwidth in the simulation of about $55 \mathrm{~nm}$ (a $4 \%$ relative bandwidth). The oscillatory features visible in the transmission region have a spectral periodicity of $10 \mathrm{~nm}$ that corresponds 


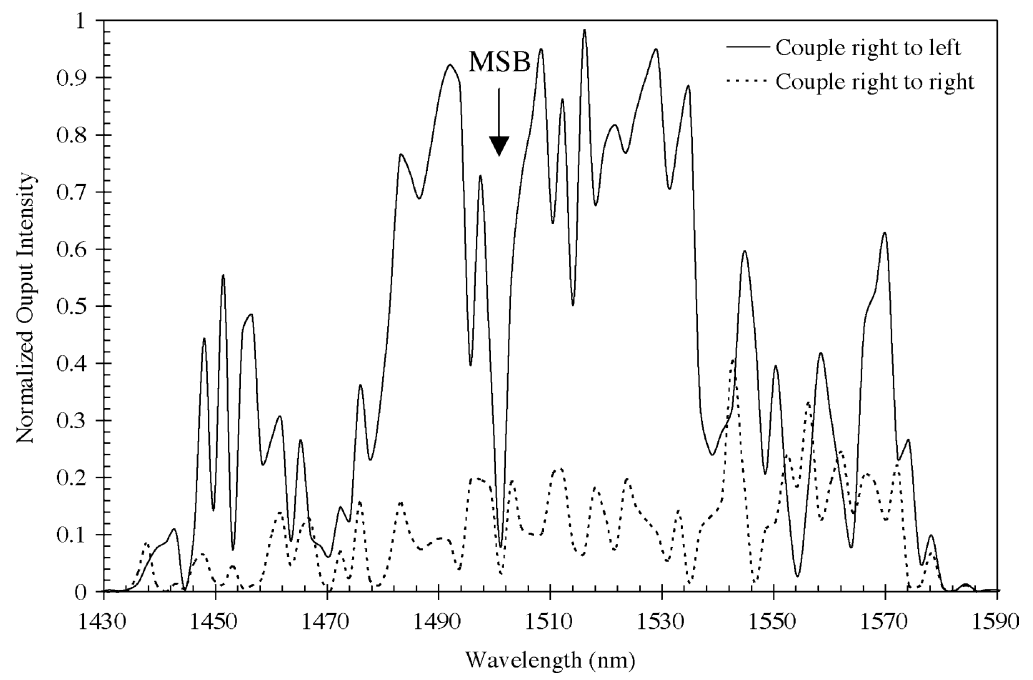

Fig. 2. Simulated transmission power spectra for the two output ports of the four-port device.

to a distance of approximately $40 \mu \mathrm{m}$, in the guide medium of the four-port coupler $\mathrm{PhC}$ structure, i.e. the periodicity is determined by the sample total length and is not due to external reflection effects.

The drop feature present in the transmission spectrum around $\lambda=1500 \mathrm{~nm}$, is expected because of the mini-stop-band (MSB) from the two periods wide (W2) channel formed at the interface of the three rows at the channel Y-junction and the direct channelto-channel coupler section. This type of behaviour has also been reported in [16] and we believe that the W2 part can be optimized to reduce mini-stop-band effects.

\section{Fabrication processes}

The device was fabricated on a silicon-on-insulator (SOI) wafer. The upper cladding of the guide structure was formed by a $200 \mathrm{~nm}$ thick layer of deposited plasma enhanced chemical vapour deposition (PECVD) $\mathrm{SiO}_{2}$, while the core was a $340 \mathrm{~nm}$ thick layer of silicon and the lower cladding was a $3 \mu \mathrm{m}$ thick layer of $\mathrm{SiO}_{2}$ on a silicon substrate.

The PhC pattern was then written on the sample wafer using direct-write electron-beam lithography (EBL). Two stages of reactive ion-etching were also required to transfer the final pattern into the substrate.
The sample was thinned to provide good cleaved facets, necessary for in-coupling and out-coupling of the light through the measured structure in the end-fire coupling method measurements. Fig. 3 shows scanning electron micrographs with details of the device fabricated in SOI material.

The fabricated holes diameters were $50 \mathrm{~nm}$ smaller than the designed size, which may be due to the smaller exposure dose used to transfer the pattern generated by EBL. Use of a higher exposure dose can cause over-exposure of the holes in the areas that require a $50 \%$ filling factor and can also be due to charging problems in the top and lower cladding silica layers. This problem can be avoided by using a thin film of metal on top of the resist, as reported in [19] and could produce holes that are closer to perfectly circular.

The wet steam thermal-oxidation process was chosen to enlarge the size of the holes by $50 \mathrm{~nm}$ in diameter by growing a thin layer of $\mathrm{SiO}_{2}$ thermally on the silicon core. This $\mathrm{SiO}_{2}$ layer can be removed after the thermal oxidation by wet etching using HF. The wet process produced a good quality thin film oxide at a fast rate (usually $3 \mathrm{~nm} / \mathrm{min}$ ) and also helps to improve the shape and the sidewall of the holes and waveguide used to couple light into the device.

On the other hand, the thin layer of thermally grown $\mathrm{SiO}_{2}$ decreases the effective refractive index, 


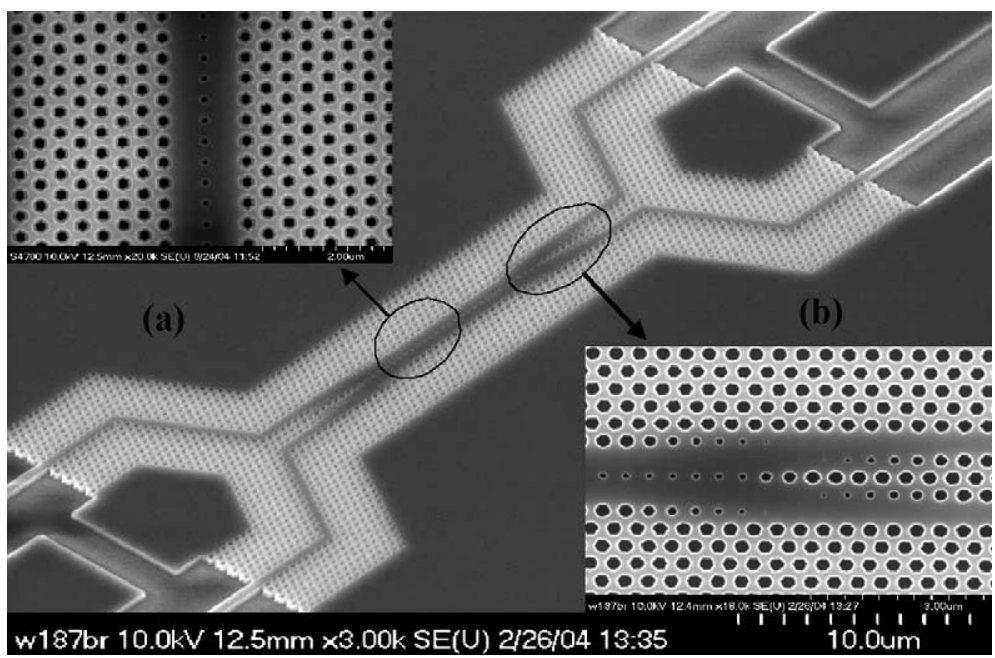

Fig. 3. Scanning electron micrographs of the four-port device fabricated on SOI material: (a) details of the small holes part of the 27 periods directional coupler; (b) details of the modified splitter using a mode conversion region showing taper holes.

changing the operational bandwidth of the device: this phenomenon can help also in tuning the device, if necessary, due to fabrication tolerances-but needs to be used carefully because the beneficial effects of forming the $\mathrm{SiO}_{2}$ layer can be over-taken by the behaviour resulting from enlargement of the hole size. The wet steam thermal oxidation is one of the processes that can be used to overcome the fabrication problems related to the size of the $\mathrm{PhC}$ holes produced in SOI material.

\section{Experimental results}

The device was characterized using TE-polarized light from a tunable laser source over the range from

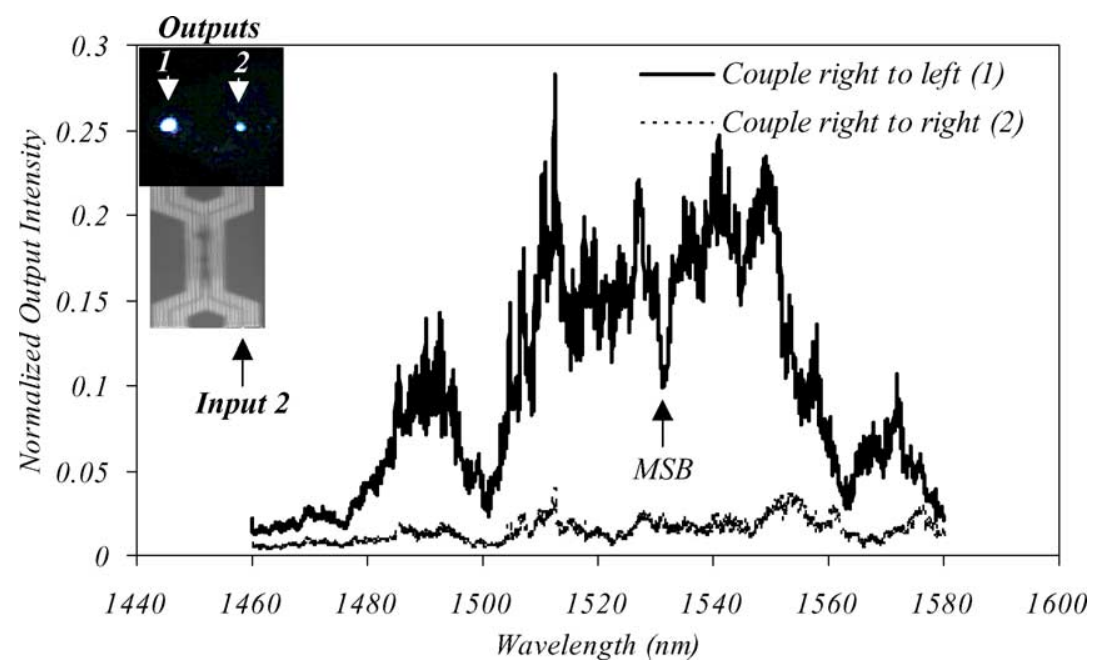

Fig. 4. Transmission spectra: the dashed line shows the output of the direct channel waveguide, output 2 (light launched in the input 2 ) and the bold line shows the output for the coupled channel waveguide, output 1 (light launched in the input 2). Inset, infrared camera image of the two output at $\lambda=1520 \mathrm{~nm}$. 
1460 to $1580 \mathrm{~nm}$. Light was coupled into and out of the waveguides using an end-fire approach. Fig. 4 shows the transmission characteristics measured on the device fabricated in SOI material. The dashed line is the transmission straight through to output 2 when light is coupled into input 2 and the infrared image (inset) shows that a small amount of light still remains.

The bold line is the transmission through to output 1 with light coupled into input 2 . The infrared image shows a more intense spot and the difference in the total transmission is shown spectrally in the curves of Fig. 4. A Vidicon infrared camera is used to facilitate the alignment of the stage and also helps to estimate the intensity profile of the out-coupling waveguide, inset in Fig. 4.

Measurement and simulation show close agreement, with a measured transmission bandwidth of approximately $55 \mathrm{~nm}$ over the range $\lambda=1505$ $1560 \mathrm{~nm}$. The modest shift in the curves of about $25 \mathrm{~nm}$ required to obtain close agreement is probably due to the changes in the refractive index caused by the $\mathrm{SiO}_{2}$ grown during the thermal-oxidation process and also could be due to the difference in the effective index used in the simulation.

The drop feature is also present in the measured device, confirming the mini-stop-band (MSB) behaviour associated with the mode conversion region (PhC waveguide W2). The re-designed Y-junction avoids the incoming signal resonating and scattering back into other input to the Y-junction and, combined with the shifted holes in the bend region, contributes to enhancement of the transmission. The two measured 'side-lobe' features were also fairly closely reproduced in the simulation. The experimental result was normalized with respect to a $\mathrm{PhC}$ waveguide with the same total length and the total additional loss expected in the device is about $-8 \mathrm{~dB}$.

\section{Conclusions}

This paper has presented work on a four-port coupler device based on 2D photonic crystal structures fabricated in silicon-on-insulator material, using W1 channel waveguides oriented along the $\mathrm{PhC} \Gamma K$ direction. A device operating at $\lambda=1550 \mathrm{~nm}$ has been successfully fabricated and measured. The measured and simulated transmission spectra have been shown to be in good agreement, with a modest shift in the main pass-band attributable to the change in the effective refractive index caused by the thermaloxidation process used to enlarge the fabricated holes.

The structure was fabricated using a combination of direct-write electron-beam lithography (EBL) and dry-etch processing. The device has demonstrated its potential for use in future low cost and compact optical switches that could be produced, e.g. by incorporating an appropriately designed and located heater electrode on the photonic crystal structure, in order to use the thermo-optic effect as a mechanism for modifying the phase-shift in the coupler, as related in the authors' previous paper [5] and, equally importantly, modifying the refractive index distribution across the coupler section. Other switching mechanisms, such as the Pockels electro-optic effect, could also be used, in a suitable material system.

\section{Acknowledgements}

One author (Edilson Camargo) wishes to thank BAe Systems/UK and Fundação Coordenação de Aperfeiçoamento de Pessoal de Nível Superior-CAPES/ Brazil for their support and sponsorship for this work, as part of the author's Ph.D. programme at the University of Glasgow. H.M.H. Chong was supported by the European Community IST project PICCO during part of the period in which this work was carried out.

\section{References}

[1] M. Lončar, J. Vučković, A. Scherer, Methods for controlling positions of guided modes of photonic-crystal waveguides, J. Opt. Soc. Am. 18 (2001) 1362-13368.

[2] I. Ntakis, P. Pottier, E. Camargo, R.M. De La Rue, Efficient transmission of $60^{\circ}$ photonic crystal bend by waveguide width tuning, in: Proceedings of the 15th Meeting of the IEEE Laser and Electro-Optics Society (LEOS), 10-14 November, Glasgow, UK, 2002.

[3] I. Ntakis, P. Pottier, R.M. De La Rue, Optimization of transmission properties of two-dimensional photonic crystal channel waveguide bends through local lattice deformation, J. Appl. Phys. 96 (1) (2004) 12-18.

[4] E.A. Camargo, A.S. Jugessur, I. Ntakis, R.M. De La Rue, Photonic crystal waveguide Mach-Zehnder structures for thermo-optic switching, in: G.C. Righini (Ed.), Integrated Optical Devices: Fabrication and Testing, Proc. SPIE 4944 (2003) 376-381. 
[5] E.A. Camargo, H.M.H. Chong, R.M. De La Rue, 2D photonic crystal thermo-optic switch based on AlGaAs/GaAs epitaxial structure, Optics Express 12 (2004) 588-592.

[6] E.A. Camargo, R.M. De La Rue, Photonic crystal beam splitter based on AlGaAs/GaAs epitaxial structure, in: Proceedings of the OSA-Topical Meetings of Integrated Photonics Research, June, San Francisco, USA, 2004.

[7] M. Thorhauge, L.H. Frandsen, P.I. Borel, Efficient photonic crystal directional couplers, Optics Lett. 28 (17) (2003) 15251527.

[8] Y. Sugimoto, Y. Tanaka, N. Ikeda, T. Yang, H. Nakamura, K. Asakawa, K. Inove, T. Maruyama, K. Miyashita, K. Ishida, Y. Watanabe, Design, fabrication and characterization of coupling-strength-controlled directional coupler based on twodimensional photonic-crystal slab waveguides, Appl. Phys. Lett. 83 (16) (2003) 3236-3238.

[9] J. Zimmermann, M. Kamp, A. Forchel, R. März, Photonic crystal waveguide directional couplers as wavelength selective optical filters, Optics Commun. 203 (2004) 387-392.

[10] M. Qiu, M. Mulot, M. Swillo, S. Anand, B. Jaskorzynska, A. Karlsson, M. Kamp, A. Forchel, Photonic crystal optical filter based on contra-directional waveguide coupling, Appl. Phys. Lett. 83 (25) (2003) 5121-5123.

[11] H.P. Zappe, Introduction to Semiconductor Integrated Optics, Artech House Inc., 1995.

[12] S. Somekh, E. Garmire, A. Yariv, H.L. Garvin, R.G. Hunsperger, Channel optical waveguide directional couplers, Appl. Phys. Lett. 22 (1973) 46-47.
[13] F. Cuesta-Soto, A. Martinez, J. Garcia, F. Ramos, P. Sanchis, J. Blasco, J. Marti, All-optical switching structure based on a photonic crystal directional coupler, Optics Express 12 (1) (2004) 161-167.

[14] A. Martinez, F. Cuesta, J. Marti, Ultrashort 2D photonic crystal directional couplers, IEEE Photon. Technol. Lett. 15 (2003) 694-696.

[15] M. Palamaru, Ph. Lalanne, Photonic crystal waveguides: outof-plane losses and adiabatic modal conversion, Appl. Phys. Lett. 78 (2001) 1466-1469.

[16] $\mathrm{Ph}$. Lalanne, A. Talneau, Modal conversion with artificial materials for photonic-crystal waveguides, Optics Express 10 (8) (2002) 354-359.

[17] A. Talneau, M. Mulot, S. Anand, Ph. Lalanne, Compound cavity measurement of transmission and reflection of a tapered single-line photonic-crystal waveguide, Appl. Phys. Lett. 82 (16) (2003) 2577-2579.

[18] A. Talneau, Ph. Lalanne, M. Agio, C.M. Soukoulis, Lowreflection photonic-crystal taper for efficient coupling between guide sections of arbitrary width, Optics Lett. 27 (17) (2002) 1522-1524.

[19] S. Yliniemi, T. Aalto, P. Heimala, P. Pekko, K. Jefimovs, J. Simonem, T. Uusitupa, Fabrication of photonic crystal waveguide elements on SOI, in: G.C. Righini (Ed.), Integrated Optical Devices: Fabrication and Testing, Proc. SPIE 4944 (2003) 376-381. 